OHSTPY-HEP-T-13-002

LPSC-13183

CETUP2013-006

\title{
LHC Phenomenology of SO(10) Models with Yukawa Unification
}

\author{
Archana Anandakrishnan ${ }^{\dagger}$, B. Charles Bryant ${ }^{\dagger}$, Stuart Raby ${ }^{\dagger}$, and Akın Wingerter ${ }^{\ddagger}$ \\ ${ }^{\dagger}$ Department of Physics, The Ohio State University, \\ 191 W. Woodruff Ave, Columbus, OH 43210, USA \\ ${ }^{\ddagger}$ Laboratoire de Physique Subatomique et de Cosmologie, \\ UJF Grenoble 1, CNRS/IN2P3, INPG, \\ 53 Avenue des Martyrs, F-38026 Grenoble, France
}

September 10, 2021

\begin{abstract}
In this paper we study an SO(10) SUSY GUT with Yukawa unification for the third generation. We perform a global $\chi^{2}$ analysis given to obtain the GUT boundary conditions consistent with 11 low energy observables, including the top, bottom and tau masses. We assume a universal mass, $m_{16}$, for squarks and sleptons and a universal gaugino mass, $M_{1 / 2}$. We then analyze the phenomenological consequences for the LHC for 15 benchmark models with fixed $m_{16}=20 \mathrm{TeV}$ and with varying values of the gluino mass. The goal of the present work is to (i) evaluate the lower bound on the gluino mass in our model coming from the most recent published data of CMS and (ii) to compare this bound with similar bounds obtained by CMS using simplified models. The bottom line is that the bounds coming from the same sign di-lepton analysis are comparable for our model and the simplified model studied assuming $\mathcal{B}\left(\tilde{g} \rightarrow t \bar{t} \tilde{\chi}_{1}^{0}\right)=100 \%$. However the bounds coming from the purely hadronic analyses for our model are $10-20 \%$ lower than obtained for the simplified models. This is due to the fact that for our models the branching ratio for the decay $\tilde{g} \rightarrow g \tilde{\chi}_{1,2}^{0}$ is significant. Thus there are significantly fewer b-jets. We find a lower bound on the gluino mass in our models with $M_{\tilde{g}} \gtrsim 1000$ $\mathrm{GeV}$. Finally, there is a theoretical upper bound on the gluino mass which increases with the value of $m_{16}$. For $m_{16} \leq 30 \mathrm{TeV}$, the gluino mass satisfies $M_{\tilde{g}} \leq 2.8 \mathrm{TeV}$ at $90 \%$ CL. Thus, unless we further increase the amount of fine-tuning, we expect gluinos to be discovered at LHC 14.
\end{abstract}




\section{Introduction}

In a previous paper, [1], some of the present authors performed a global $\chi^{2}$ analysis on the DermisekRaby [DR] model $[2,3]$. The DR model has $S O(10)$ Yukawa unification for the third family of quarks and leptons and a hierarchical set of Yukawa matrices for the two lighter families which is determined by a hierarchical breaking of a $D_{3}$ family symmetry. This model has 24 arbitrary parameters $(22$ gauge, Yukawa and soft SUSY breaking parameters defined at the GUT scale with two additional parameters, $\mu, \tan \beta$ fixed at the weak scale), see Tab. 1 . With a $\chi^{2}$ function including 36 low energy observables we found the best fit with $\chi^{2} /$ d.o.f. $=2$ for a value of the universal squark and slepton mass parameter, $m_{16}=20 \mathrm{TeV}$ and a lower bound on the gluino mass, $M_{\tilde{g}}=850 \mathrm{GeV}$, imposed via a $\chi^{2}$ penalty. In the previous paper it was also demonstrated that the SUSY particle spectrum was constrained solely by a third family analysis. It is this analysis we use in the present paper.

Note, the gluino mass prefers to be even smaller than $850 \mathrm{GeV}$, but a lower bound was imposed to be roughly consistent with recent LHC SUSY search bounds. The goal of this paper is to find the actual limits on the SUSY spectrum consistent with Yukawa unification by directly comparing with the most recent LHC SUSY searches. The SUSY bounds which are most relevant to our SUSY spectrum are those obtained using simplified models. In particular, we find the squarks and sleptons of the first two families to have a large mass of order $m_{16}$ which we take to be between 10 and $30 \mathrm{TeV}$. The third family of squarks and sleptons, however, are significantly lighter and finally the gauginos are even lighter. Therefore, the simplified models which might come closest to constraining our results are those in which gluinos are pair produced at the LHC and then decay with $100 \%$ branching ratio $\tilde{g} \rightarrow t \bar{t} \tilde{\chi}_{1}^{0}$ or $\tilde{g} \rightarrow b \bar{b} \tilde{\chi}_{1}^{0}$. The lower bound on the gluino mass from CMS [4-6] and ATLAS [7-9] searches are of order $M_{\tilde{g}} \geq 1-1.2 \mathrm{TeV}$. In this paper we will not impose a lower bound on the gluino mass by hand, as we had done in our previous analysis. In Fig. 1 we plot $\chi^{2}$ as a function of the gluino mass in a third family analysis. Since $M_{1 / 2}$ and $m_{16}$ are fixed at the GUT scale, there are 9 arbitrary parameters and 11 low energy observables or 2 degrees of freedom. The gluino mass wants to be low with the best fit at $M_{\tilde{g}} \simeq 600 \mathrm{GeV}$. We also see that $\chi^{2}$ increases as the gluino mass increases. Thus as discussed in [1] there is an upper bound on the gluino mass such that at $90 \%$ CL we have $M_{\tilde{g}} \lesssim 2.0 \mathrm{TeV} .{ }^{1}$ In fact, we shall show that the $68 \%$ C.L. interval is not totally excluded by LHC searches. We find that gluinos are allowed with mass approximately $20 \%$ smaller than presently excluded using some simplified model analyses. Nevertheless we find the strongest constraint comes from the same sign di-lepton analysis and the hadronic $\Delta \phi$ analysis which both give $M_{\tilde{g}} \gtrsim 1 \mathrm{TeV}$.

The paper is organized as follows. In Section 2 we describe the model and the benchmark points used in the present analysis. Our analysis uses several of the SUSY tools in the literature, such as Prospino, Pythia, Delphes, SDecay and ROOT, in addition to the code Maton, developed by Dermisek and revised by Anandakrishnan and Wingerter to be applicable to the hierarchy of the low energy SUSY scales as discussed in Section 3. We calculate the constraints on the gluino mass using the same sign di-lepton and hadronic analyses of CMS. These are the most relevant ones for our model, since there is sufficient information to abstract the 95\% C.L. upper limit on the number of SUSY events allowed in each signal region. The analysis using the CMS data for same sign di-leptons is found in Section 4 and jets plus missing $E_{T}$ is in Section 5. Finally a summary of our results and

\footnotetext{
${ }^{1}$ This upper bound depends on the fact that $m_{16}=20 \mathrm{TeV}$. For larger $m_{16}$ the upper bound on the gluino mass increases (See Appendix A for more details).
} 
conclusions is given in Section 6 .

\section{Benchmark Models}

In Ref. [1], it was found that the soft SUSY spectrum was completely determined by fitting 11 low energy observables, $M_{W}, M_{Z}, G_{F}, \alpha_{e m}^{-1}, \alpha_{s}\left(M_{Z}\right), M_{t}, m_{b}\left(m_{b}\right), M_{\tau}, \mathcal{B}\left(B \rightarrow X_{s} \gamma\right), \mathcal{B}\left(B_{s} \rightarrow \mu^{+} \mu^{-}\right)$, and the lightest Higgs mass, $M_{h}$. Therefore in this paper we use the third family analysis exclusively with the 11 arbitrary parameters listed in Tab. 1. The universal Yukawa coupling $\lambda$ appears in the superpotential term $\mathcal{W} \supset \lambda 16_{3} 1016_{3}$.

In the present analysis we neglect all Yukawa terms except for the third family coupling, $\lambda$, and integrate out the right-handed neutrinos at the GUT scale. One might wonder whether neglecting the off-diagonal elements of the Yukawa matrices, as considered in our three family analysis, and also not allowing the right-handed neutrinos to be integrated out at lower scale, more appropriate for the See-Saw mechanism, might effect significant deviations from the earlier paper Ref. [1]. Nevertheless, we find that within a few percent the results are the same. In the former case, however, the magnitude of the Higgs splitting parameter is all that needs adjusting, since approximately $50 \%$ of the Higgs splitting necessary for electroweak symmetry breaking is generated as a threshold effect due to the right-handed neutrinos.

\begin{tabular}{|l||c|c||c|c||}
\hline Sector & Third Family Analysis & $\#$ & Full three family Analysis & $\#$ \\
\hline gauge & $\alpha_{G}, M_{G}, \epsilon_{3}$ & 3 & $\alpha_{G}, M_{G}, \epsilon_{3}$ & 3 \\
SUSY (GUT scale) & $m_{16}, M_{1 / 2}, A_{0}, m_{H_{u}}, m_{H_{d}}$ & 5 & $m_{16}, M_{1 / 2}, A_{0}, m_{H_{u}}, m_{H_{d}}$ & 5 \\
textures & $\lambda$ & 1 & $\epsilon, \epsilon^{\prime}, \lambda, \rho, \sigma, \tilde{\epsilon}, \xi$ & 11 \\
neutrino & & 0 & $M_{R_{1}}, M_{R_{2}}, M_{R_{3}}$ & 3 \\
SUSY (EW scale) & $\tan \beta, \mu$ & 2 & $\tan \beta, \mu$ & 2 \\
\hline Total \# & \multicolumn{1}{|r|}{} & & 24 \\
\hline
\end{tabular}

Table 1: The model is defined by three gauge parameters, $\alpha_{G}, M_{G}, \epsilon_{3}$; one large Yukawa coupling, $\lambda ; 5$ SUSY parameters defined at the GUT scale, $m_{16}$ (universal scalar mass for squarks and sleptons), $M_{1 / 2}$ (universal gaugino mass), $m_{H_{u}}, m_{H_{d}}$ (up and down Higgs masses), and $A_{0}$ (universal trilinear scalar coupling); $\mu$, tan $\beta$ obtained at the weak scale by consistent electroweak symmetry breaking. The full three family model has additional off-diagonal Yukawa couplings, and includes 3 right-handed neutrino masses.

We have to emphasize, however, one important difference with respect to the previous analysis. In Ref. [1], we incorporated collider constraints by requiring that the gluino be greater than a given lower bound, i.e. while minimizing $\chi^{2}$, we introduced a penalty whenever the gluino mass was less than this lower bound. These lower bounds were taken from LHC analyses based on so-called simplified models. Although as a first guess, bounds derived using simplified models may be useful, it turns out that in practice the assumptions entering the analyses are rarely met.

From what we have discussed so far it follows that it does not make much sense to constrain the $\chi^{2}$ minimization by requiring a lower bound on the gluino mass derived from simplified models. As a consequence, we drop this requirement and present in Fig. 1 the corresponding $\chi^{2}$ as a function of the gluino mass. More specifically, of the 11 parameters defining the third-family $\mathrm{SO}(10)$ Yukawa unified model (see Tab. 1), we fix $m_{16}=20 \mathrm{TeV}$, and then minimize $\chi^{2}$ for 15 values of $M_{1 / 2}$ 


\begin{tabular}{|l|l|l|l|l|l|l|}
\hline & YUa & YUb & YUc & YUd & YUe & YUf \\
\hline$M_{1 / 2}$ & 150 & 200 & 250 & 300 & 400 & 600 \\
$\mu$ & 869 & 889 & 824 & 878 & 975 & 664 \\
\hline$\chi^{2}$ & 0.22 & 0.43 & 0.77 & 1.17 & 2.24 & 3.97 \\
\hline$M_{A}$ & 2299 & 2323 & 2262 & 2300 & 2750 & 2120 \\
$m_{\tilde{t}_{1}}$ & 3775 & 3789 & 3695 & 3728 & 3775 & 3530 \\
$m_{\tilde{b}_{1}}$ & 4633 & 4654 & 4579 & 4607 & 4628 & 4526 \\
$m_{\tilde{\tau}_{1}}$ & 7865 & 7885 & 7834 & 7806 & 7890 & 7897 \\
$m_{\tilde{\chi}_{1}^{0}}$ & 129 & 151 & 172 & 194 & 238 & 325 \\
$m_{\tilde{\chi}_{1}^{+}}$ & 263 & 303 & 342 & 382 & 461 & 587 \\
$M_{\tilde{g}}$ & 801 & 932 & 1061 & 1186 & 1430 & 1890 \\
\hline
\end{tabular}

Table 2: SUSY spectrum for six benchmark models corresponding to the third-family analysis in Ref. [1] with the qualifications detailed in the main text. All masses are in GeV. For each model, we list the 11 defining parameters in Appendix B. The scale where the first and second generation scalars are integrated out is fixed at $m_{16}=20 \mathrm{TeV}$, and the universal trilinear coupling comes out close to $A_{0}=-41 \mathrm{TeV}$ in each case as a result of the minimization. In the first column, we have assigned names to the benchmark models that we will use in the following discussion to refer to them. The third row gives the total $\chi^{2}$ and the rest of the lines are self-explanatory.

\begin{tabular}{|c|r|r|r|r|r|r|}
\hline YUa & $t b \widetilde{\chi}_{1}^{ \pm}$ & $t \bar{t} \widetilde{\chi}_{2}^{0}$ & $g \widetilde{\chi}_{2}^{0}$ & & $t \bar{t} \widetilde{\chi}_{1}^{0}$ & $b \bar{b} \widetilde{\chi}_{1}^{0}$ \\
& $52 \%$ & $22 \%$ & $19 \%$ & & $4.5 \%$ & $0.07 \%$ \\
\hline YUb & $t b \widetilde{\chi}_{1}^{ \pm}$ & $t \bar{t} \widetilde{\chi}_{2}^{0}$ & $g \widetilde{\chi}_{2}^{0}$ & $g \widetilde{\chi}_{(1,3,4)}^{0}$ & $t \bar{t} \widetilde{\chi}_{1}^{0}$ & $b \bar{b} \widetilde{\chi}_{1}^{0}$ \\
& $50 \%$ & $27 \%$ & $11 \%$ & $5.5 \%$ & $4.5 \%$ & $0.04 \%$ \\
\hline YUc & $g \widetilde{\chi}_{4}^{0}$ & $g \widetilde{\chi}_{3}^{0}$ & $t b \widetilde{\chi}_{1}^{ \pm}$ & $g \widetilde{\chi}_{2}^{0}$ & $t \bar{t} \widetilde{\chi}_{1}^{0}$ & $b \bar{b} \widetilde{\chi}_{1}^{0}$ \\
& $38 \%$ & $35 \%$ & $14 \%$ & $8 \%$ & $1.2 \%$ & $0.006 \%$ \\
\hline YUd & $g \widetilde{\chi}_{4}^{0}$ & $g \widetilde{\chi}_{3}^{0}$ & $t b \widetilde{\chi}_{(1,2)}^{ \pm}$ & $t \bar{t} \widetilde{\chi}_{2}^{0}$ & $t \bar{t} \widetilde{\chi}_{1}^{0}$ & $b \bar{b} \widetilde{\chi}_{1}^{0}$ \\
& $35 \%$ & $33 \%$ & $21 \%$ & $7 \%$ & $1 \%$ & $0.004 \%$ \\
\hline YUe & $t b \widetilde{\chi}_{2}^{ \pm}$ & $g \widetilde{\chi}_{(3,4)}^{0}$ & $t \bar{t} \widetilde{\chi}_{(2,3,4)}^{0}$ & $t b \widetilde{\chi}_{1}^{ \pm}$ & $t \bar{t} \widetilde{\chi}_{1}^{0}$ & $b \bar{b} \widetilde{\chi}_{1}^{0}$ \\
& $34 \%$ & $34 \%$ & $23 \%$ & $6 \%$ & $0.5 \%$ & $0.0002 \%$ \\
\hline YUf & $t \bar{t} \widetilde{\chi}_{4}^{0}$ & $t b \widetilde{\chi}_{2}^{ \pm}$ & $t \bar{t} \widetilde{\chi}_{3}^{0}$ & $t b \widetilde{\chi}_{1}^{ \pm}$ & $t \bar{t} \widetilde{\chi}_{1}^{0}$ & $b \bar{b} \widetilde{\chi}_{1}^{0}$ \\
& $31 \%$ & $23 \%$ & $19 \%$ & $14 \%$ & $0.25 \%$ & $0.000001 \%$ \\
\hline
\end{tabular}

Table 3: Gluino decay branching ratios into different final states for the six benchmark models given in Tab. 2. For each model, we give the dominant branching fractions. The last two columns give the branching fractions of the gluino into $t \bar{t} \widetilde{\chi}_{1}^{0}$ and $b \bar{b} \widetilde{\chi}_{1}^{0}$ which correspond to the simplified scenarios studied by the CMS analyses. These rates were calculated using SDECAY. 


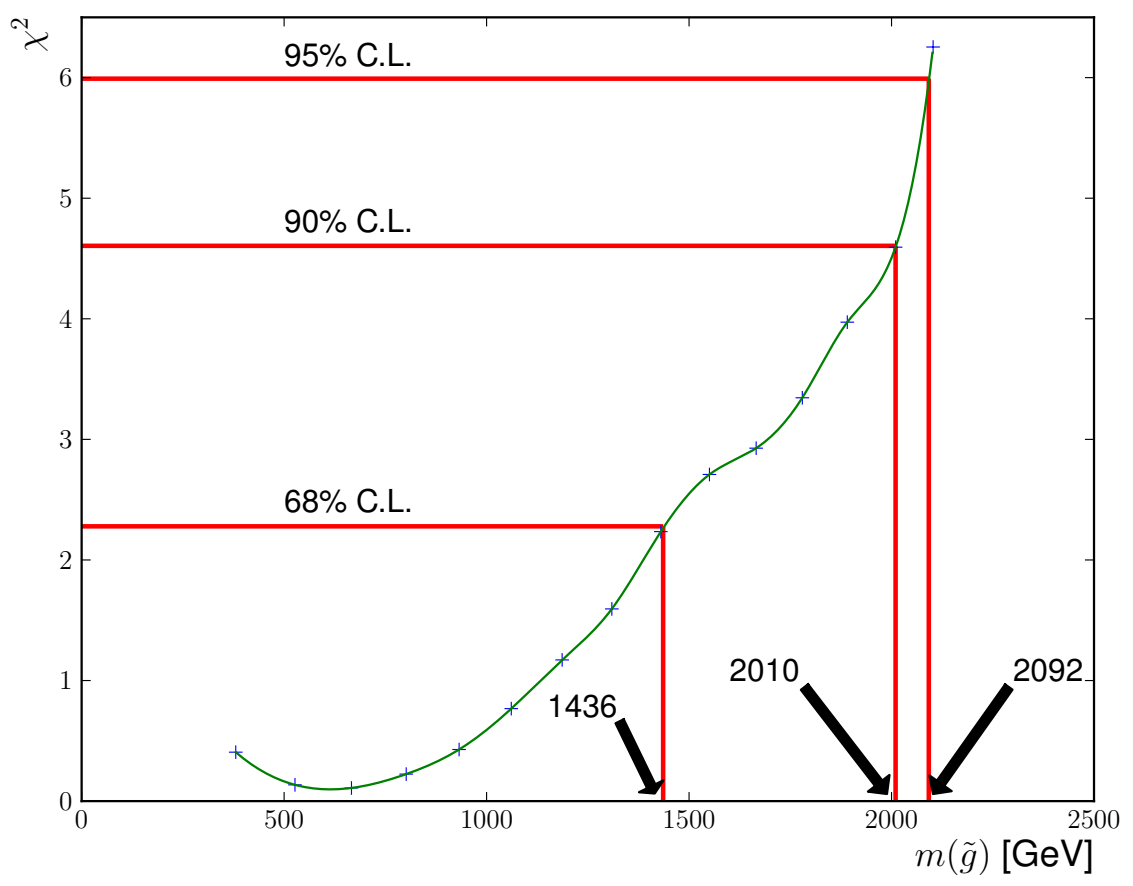

Figure 1: The $\chi^{2}$ function for fixed $m_{16}=20 \mathrm{TeV}$ and different values of $M_{1 / 2}$ corresponding to the gluino masses indicated on the $x$-axis. In minimizing $\chi^{2}$ for each point, we followed the same procedure as in Ref. [1] except that we now do not penalize for gluino masses smaller than a certain lower bound. The confidence level intervals correspond to the $\chi^{2}$ distribution with two degrees of freedom.

between 0 and $600 \mathrm{GeV}$ by varying all the other parameters. The $x$ axis in Fig. 1 is $M_{\tilde{g}}$. The data points are indicated in Fig. 1 by crosses, and we have connected them using a cubic spline interpolation to guide the eye. We calculate the $68 \%, 90 \%$ and $95 \%$ confidence level intervals using the $\chi^{2}$ distribution for 2 degrees of freedom; the corresponding values are indicated in Fig. 1 by the horizontal red lines. We find that they correspond to gluino masses of 1436, 2010, and $2092 \mathrm{GeV}$ and are currently far from being excluded even by the simplified model analyses. As benchmark models in Tab. 2 we have chosen six models along this $\chi^{2}$ curve corresponding to a range of different gluino masses.

In our models, the first and second generation squarks are heavy, and the particles in the spectrum that are accessible at the LHC are the the gluinos, the lightest neutralino and the charginos (cf. Tab. 2). The simplified models that come closest to our setup assume branching ratios of $\mathcal{B}\left(\widetilde{g} \rightarrow t \bar{t} \widetilde{\chi}_{1}^{0}\right)=100 \%$ or $\mathcal{B}\left(\widetilde{g} \rightarrow b \bar{b} \widetilde{\chi}_{1}^{0}\right)=100 \%$. For the benchmark models (Tab. 2) the decay branching ratios for the gluino are given in Tab. 3 . These clearly do not match the decay branching ratios for any simplified model.

Fortunately, the ATLAS and CMS collaborations provide in many cases the raw data necessary to reinterpret the searches for new physics in the context of more elaborate models. E.g. the model A1 in Ref. [5] assumes $\mathcal{B}\left(\widetilde{g} \rightarrow t \bar{t} \widetilde{\chi}_{1}^{0}\right)=100 \%$, but the expected number of SM events in each signal region (cmp. Tab. 2 in the same publication) allows us to derive exclusion bounds for our model on the basis of not having observed a signal. This is in essence what we are doing in this paper. We 


\begin{tabular}{|c|c|c|c|}
\hline Analysis & Luminosity & Signal Region & Reference \\
\hline SS di-lepton & 10.5 & $\begin{aligned} N_{\text {jet }} & \geq 4, N_{\mathrm{b}-\text { jet }} \geq 2, \\
E_{T}^{\text {miss }} & >120, H_{T}>200\end{aligned}$ & {$[5]$} \\
\hline $\begin{array}{c}\alpha_{T} \text { analysis (for Simplified models) } \\
\text { (for the benchmark models) }\end{array}$ & 11.7 & $\begin{array}{c}N_{\text {jet }} \geq 4, N_{\text {b-jet }}=3, H_{T}>875 \\
N_{\text {jet }} \geq 4, N_{\text {b-jet }}=2,775<H_{T}<875\end{array}$ & [6] \\
\hline$\Delta \phi$ analysis & 19.4 & $N_{\mathrm{b}-\text { jet }} \geq 3, E_{T}^{\mathrm{miss}}>350, H_{T}>1000$ & {$[4]$} \\
\hline
\end{tabular}

Table 4: The most constraining signal region for each of the analyses studied in this work. All energies are in units of $\mathrm{GeV}$ and luminosity in $\mathrm{fb}^{-1}$.

will now move on to explain the procedure how to derive bounds for these models from the recent CMS analyses.

\section{Procedure}

For clarity, we have summarized the analysis chain in Fig. 3 on the following page. As mentioned before, each benchmark model is defined in terms of 11 parameters which we list for reference in Tab. 1. We use the spectrum generator maton originally written by R. Dermíšek to find the lowenergy spectrum which is then written to a file in the SUSY Les Houches Accord (SLHA) format. ${ }^{2}$ We emphasize that maton has been modified to take into account the decoupling of the first two families of squarks and sleptons. In addition, we are greatful to P. Slavich who has provided us with the code to calculate the light Higgs mass. Finally, we have compared the output SUSY spectrum of maton with SoftSUSY and the results agree to within several percent.
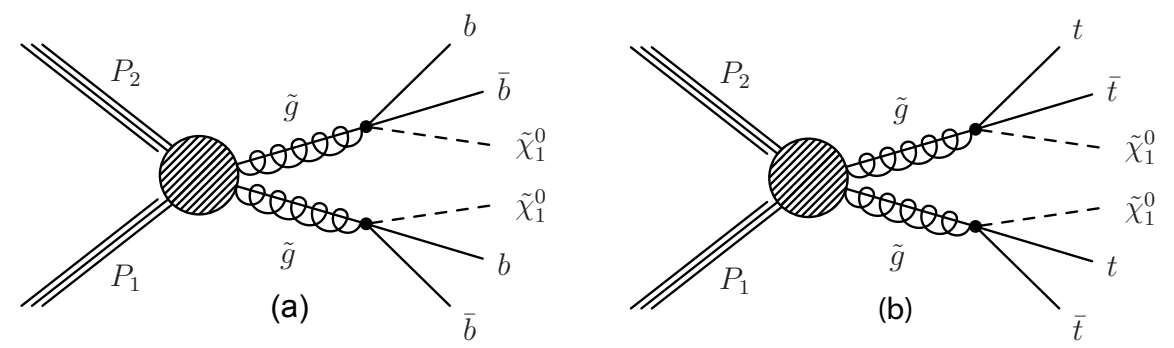

Figure 2: Feynman diagrams for the simplified SUSY scenarios (taken from Ref. [4]), T1bbbb (left) and T1tttt (right). T1bbbb (T1ttt) assumes a gluino branching ratio of $100 \%$ to $b \bar{b} \tilde{\chi}_{1}^{0}\left(t \bar{t} \tilde{\chi}_{1}^{0}\right)$.

Next we use the the output from maton as input for the program SDECAY 1.3 [11] which calculates the decay widths and branching ratios for all the SUSY particles in the spectrum. ${ }^{3}$ The output is also in SLHA format and contains along with the decay table also the spectrum that was calculated in the previous step, because this information is needed for the next steps in the analysis.

\footnotetext{
${ }^{2}$ Note that storing the spectrum in a form that is accessible by other programs is absolutely crucial for our analysis, since the different components in the work chain need to communicate with each other in order to produce data that can be directly compared to experiment.

${ }^{3}$ The gluino decay rates at large $\tan \beta$ were calculated in Refs. [12-14].
} 


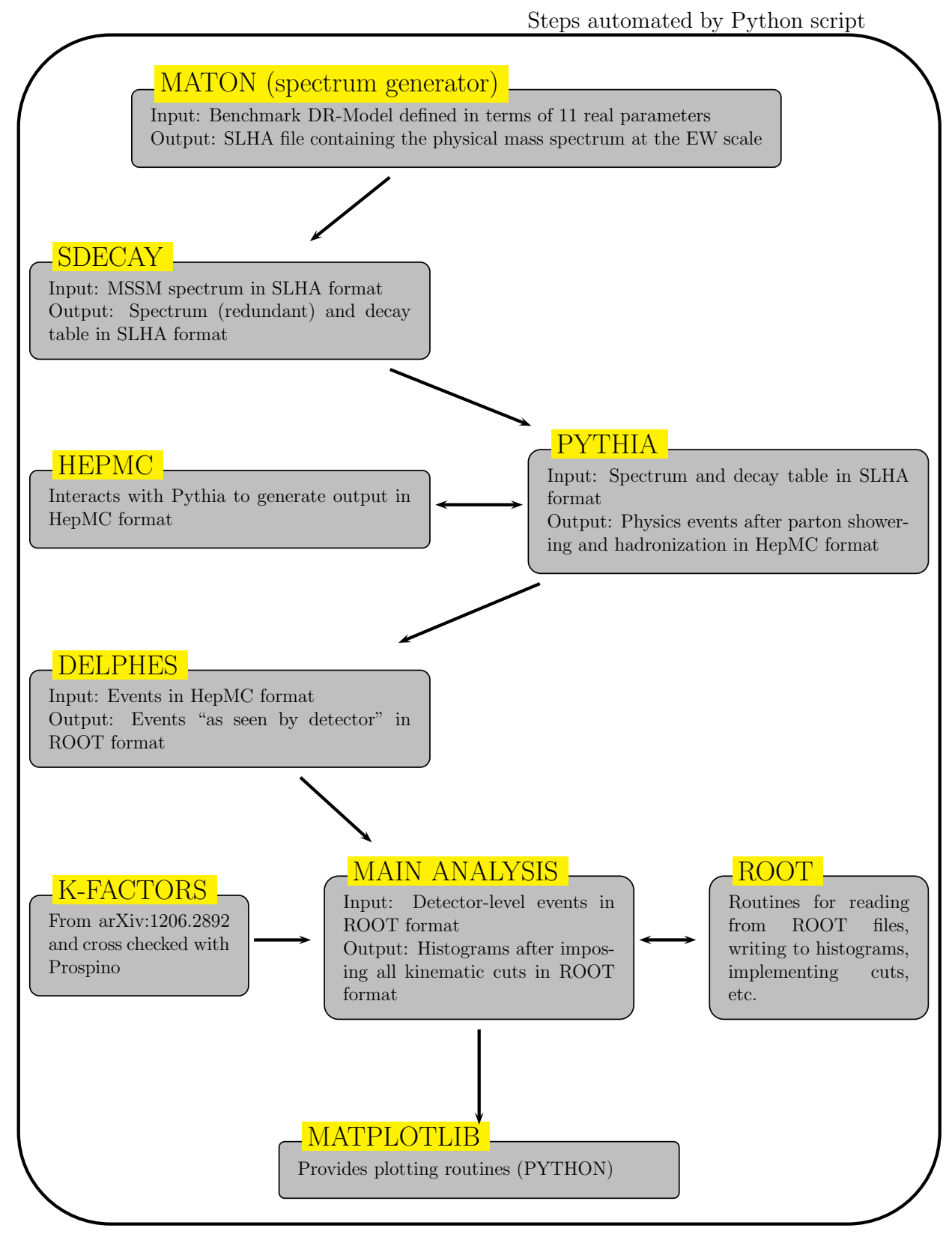

Figure 3: The flowchart illustrates the analysis chain for each benchmark model. The K-Factors are gotten from Ref. [10].

For event generation, we use PYTHIA 8.175 [15]. We have written a main program for PYTHIA that reads in all its parameters from a so-called "card file" and writes the events to a file in HepMC format $^{4}$ [16]. Our card file is identical with main24.cmnd that comes with PYTHIA as a template with the following changes indicated in Listing 1.

\footnotetext{
${ }^{4}$ We are using HepMC 2.06.09.
} 


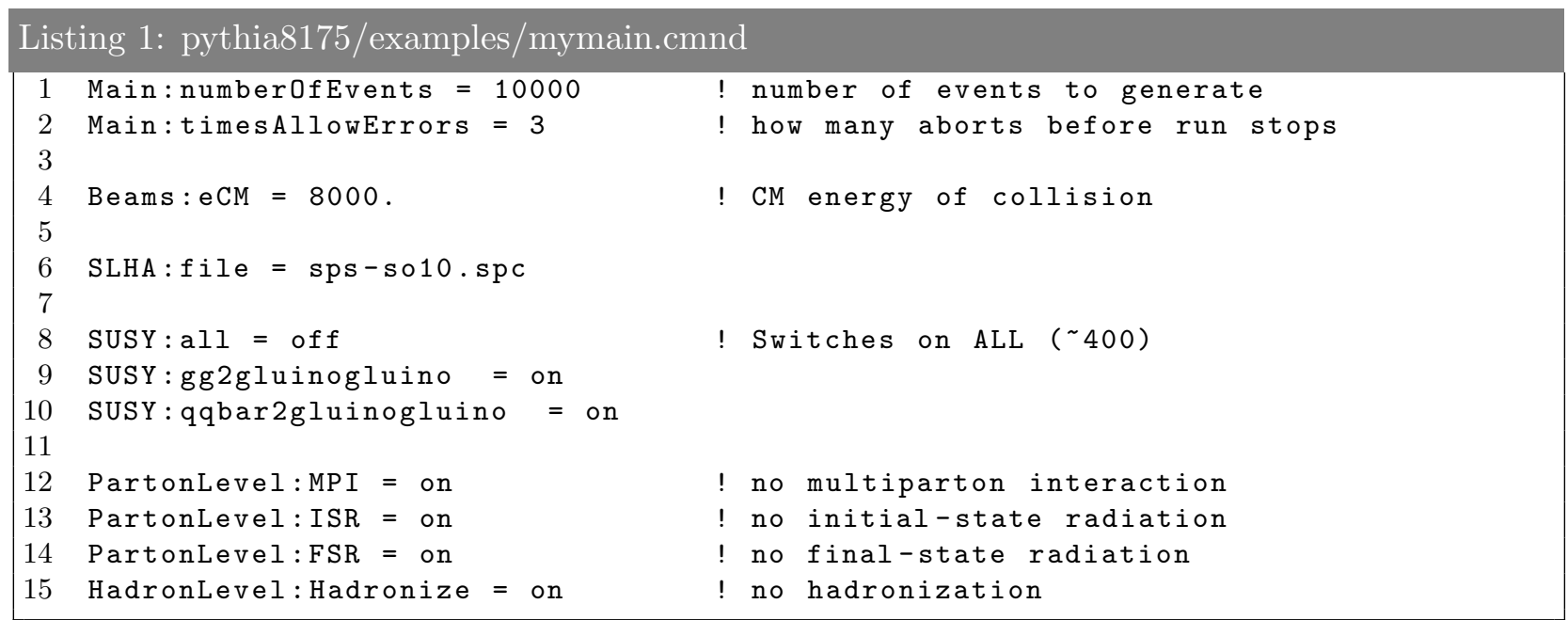

The card file is self-explanatory, and we just mention that we generate 10,000 events (line 1), and in line 6 of 1 we are reading in the decay file that we had generated in the previous step. Also, note that we have switched off all SUSY processes except $g g \rightarrow \widetilde{g} \widetilde{g}$ and $q \bar{q} \rightarrow \widetilde{g} \widetilde{g}$, since the only light particles in the spectrum are the gauginos, and the neutralinos and charginos will not contribute to the event signatures that we will later consider for the analyses. Moreover, the electroweak processes have much smaller cross sections and can also be neglected.

For the detector simulation, we use Delphes 3.0.9[17]. Compiling and running the code is straightforward, and the only file that needs to be adapted to the respective analysis is the Delphes card that chooses the detector we want to simulate (ATLAS or CMS) and sets various parameters like the b-tagging efficiency, etc. Since all these settings depend on the analysis under consideration, we will give the details in the corresponding sections further below. From the output of PYTHIA, Delphes produces a file that contains the events "as seen by the detector", i.e. particle tracks, transverse momentum $p_{T}$, missing transverse energy $E_{T}$, etc. The result is saved as a ROOT tree [18].

We now come to the main part of our analysis. Our dedicated $\mathrm{C}++$ code reads in the ROOT file and implements the event selection ${ }^{5}$ for the respective analysis. We generate the $\mathrm{C}++$ classes necessary to read in the events from the ROOT file automatically ${ }^{6}$ by the lines given in Listing 2 (to be run in ROOT).

\section{Listing 2: root/SO10-pheno/create_Analysis_class.C}

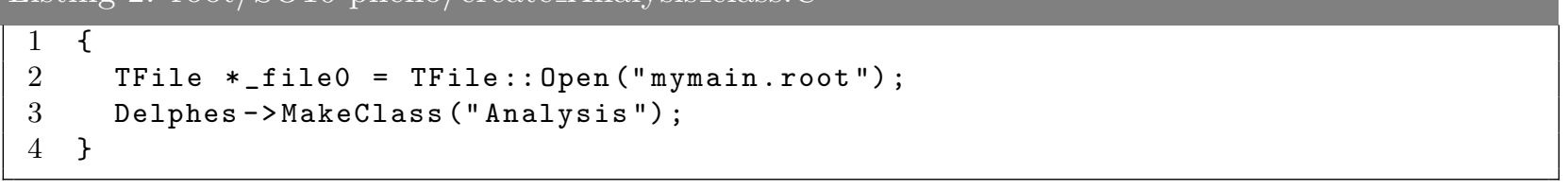

The main program implements the cuts and depends on the specific analysis at hand, and we will give some more details in the following sections. The output of the analysis (number of events

\footnotetext{
${ }^{5}$ We are using ROOT v5-28-00-patches@42209.

${ }^{6}$ We should mention that to obtain a program that compiles, one needs to include the files TRef.h and TRefArray.h in the header file that is automatically created by Listing 2 by hand; this seems to be a bug in ROOT v5-28.
} 
passing the cuts, cross sections, histograms, etc.) is stored in the form of text and ROOT files. Note that for comparing our events to experiment, we need to rescale the number of events to the luminosity of the respective analysis and correct for the leading-order gluino production cross section in PYTHIA by using $K$-factors that we obtained from Ref. [10]. We have compared these $K$-factors with what we get from Prospino 2.1.[19], and as expected we find excellent agreement. Finally, we use matplotlib 1.0.1. [20] and Python 2.7.1. to visualize the results.

\section{Same Sign Di-lepton Analysis}

We consider the 15 benchmark models corresponding to different gluino masses (Tab. 2 lists 6 of them) and investigate their discovery potential at LHC. We start with Ref. [5] that performs a search for new physics in same-sign di-lepton events with at least 2 b-jets, and concentrate on the signal region 3 (SR3) where the SM background is lowest ${ }^{7}$. For the cuts defining SR3, see Tab. 4 on page 5 .

The parameters regarding the event generation (PYTHIA) are unchanged. For the detector simulation, however, we need to adapt the Delphes card to the current analysis. In particular, in the card file delphes_card_CMS.tcl we replace the default b-tagging efficiency by the one given in Ref. [5], p. 6 .

In the main analysis, we implement all cuts and other event selection criteria that correspond to SR3 and that are described in detail in Ref. [5]. We store the number of events passing all cuts, the total gluino production cross section, and its error in a text file. Before we can plot these events and compare them to the expected SM background, we need to rescale our 10,000 gluino events to the integrated luminosity of $10.5 \mathrm{fb}^{-1}$ of events underlying the current analysis.

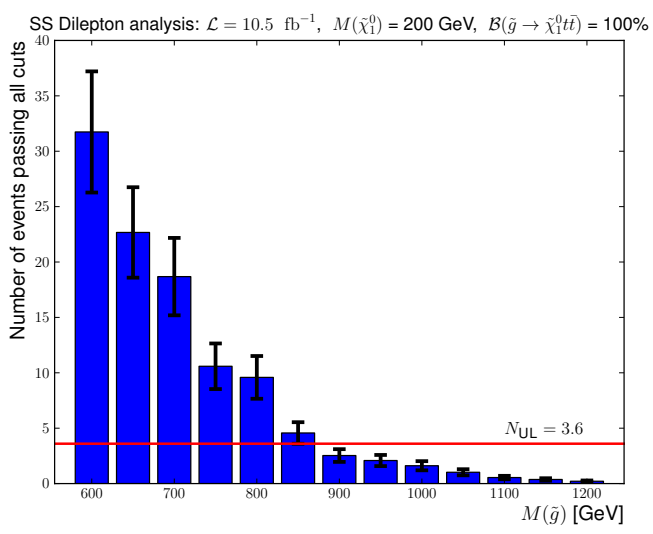

(a) Validation.

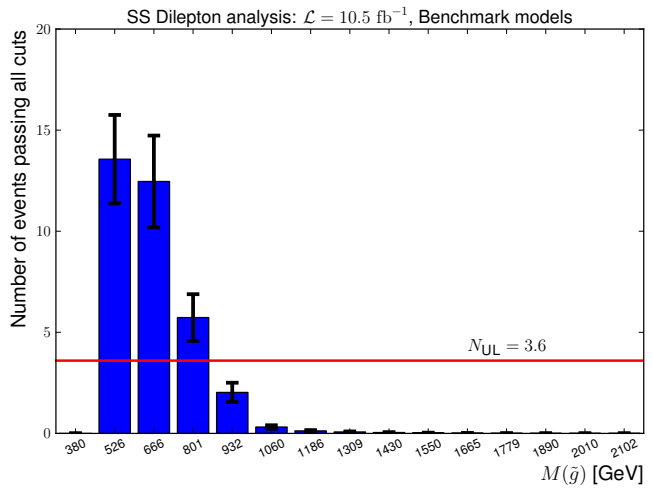

(b) Benchmark

Figure 4: The number of events passing all cuts corresponding to signal region 3 (SR3) for the simplified model A1 [5] for fixed $M_{\widetilde{\chi}_{1}^{0}}=200 \mathrm{GeV}$ (left panel) and for the benchmark models (right panel). The label on the $x$-axis denotes the gluino mass $M_{\widetilde{g}}$. The red horizontal line shows the upper limit on events from new physics at 95\% CL, and the error bars are derived from the uncertainty in the $K$-factors.

To set limits on the neutralino and gluino masses, Ref. [5] assumes a set of simplified models. The one that comes closest to our case is Model A1 (cmp. Fig. 2(b) on page 5). To validate our

\footnotetext{
${ }^{7}$ We have checked that the constraints on our models from the other signal regions are weaker.
} 
analysis, i.e. to check whether our analysis can reproduce the experimental one within reasonable error margins, we retraced the steps leading to Fig. 4 of Ref. [5]. To that end, we started out with a template SLHA file, set $\mathcal{B}\left(\widetilde{g} \rightarrow \widetilde{\chi}_{1}^{0} t \bar{t}\right)=100 \%$ and scanned over a range of gluino and neutralino masses. We exclude a model (given by a pair of gluino and neutralino masses), if the event count is greater than the 95\% CL upper limit on events from new physics (horizontal red line). In Fig. 4(a), we present a representative plot corresponding to a constant $M_{\widetilde{\chi}_{1}^{0}}=200 \mathrm{GeV}$ and read off that values of $M_{\widetilde{g}}<875 \pm 25 \mathrm{GeV}$ are excluded. The agreement between Fig. 4(a) and Fig. 4 of Ref. [5] (which gives a lower limit on the gluino mass of $\approx 1000 \pm 30 \mathrm{GeV}$ ) is well within $20 \%$, which is the expected precision that can be achieved discounting for the fact that we do not have access to the full array of experimental tools used by the ATLAS and CMS collaborations (in particular the detector simulation). In Fig. 4(b) we present the result of our analysis for the benchmark models. As is clear from the plot, models with $M_{\tilde{g}} \gtrsim 850 \mathrm{GeV}$ yield event numbers that are below the $95 \%$ upper limit allowed from models of new physics and are hence not excluded. Note that SR3 is not sensitive to very small gluino masses, but these are nevertheless excluded by other signal regions. We conclude that in this case, the simplified model captures the main features of the benchmark models and thus leads to similar collider bounds or from Ref. [5] we have $M_{\tilde{g}}>1000 \pm 30 \mathrm{GeV}$.

\section{$5 \quad$ Hadronic Analyses}

\section{$5.1 \alpha_{T}$ Analysis}

The CMS search in Ref. [6] looks for final states with multiple b-jets and missing transverse energy using a parameter $\alpha_{T}$, on which we briefly elaborate below, and interprets the results in the context of simplified models where one assumes only one decay channel, respectively, given by the Feynman diagrams in Fig. 2. The cuts in this search require large $E_{T}$ and $H_{T}$ for the jets, no isolated electrons, muons, or photons, and a cut on $\alpha_{T}$. This search was performed at $\sqrt{s}=8 \mathrm{TeV}$ and used $11.7 \mathrm{fb}^{-1}$ of data. The analysis considers only jets with $E_{T}>50 \mathrm{GeV}$ and $|\eta|<3.0$ and bins the events according to $H_{T}$ and the number of jets and b-jets. Binning details can be found in Tab. 4 of Ref. [6].

The parameter $\alpha_{T}$ is used to reject events with either little $\mathbb{E}_{T}$ or a transverse energy mismeasurement. Furthermore, it is sensitive to final states containing large, genuine $\mathbb{E}_{T}$. In a dijet system, $\alpha_{T}$ is defined as the ratio of the softer jet's transverse energy to the transverse mass of the dijet system. For events with more than two jets, a pseudo-dijet system can be formed so that $\alpha_{T}$ may still be employed. For an event in which the two jets are back-to-back, have equal transverse energy, and have momenta that are large compared to their respective masses, $\alpha_{T}$ has a value of 0.5 . In the same limit, if the two jets are back-to-back but are measured to have different transverse energies, then $\alpha_{T}$ will be less than 0.5. Events in which the two jets are not back-to-back and large, genuine $\mathscr{E}_{T}$ is present, then $\alpha_{T}$ will be much larger than 0.5. We therefore require $\alpha_{T}>0.55$.

The Delphes CMS card is modified to contain the electron, muon, and photon isolation criteria given in Sec. 4 of Ref. [6]. In the same section, the paper states that the b-tagging efficiency is $60-70 \%$ and $p_{T}$-dependent. We therefore use the same b-tagging efficiency as for the di-lepton analysis. To validate our procedure for this analysis, we calculate bounds on the T1bbbb and T1tttt simplified models (Fig. 2) assuming $100 \%$ branching ratios to $\tilde{\chi}_{1}^{0} b \bar{b}$ and $\tilde{\chi}_{1}^{0} t \bar{t}$, respectively, using the 

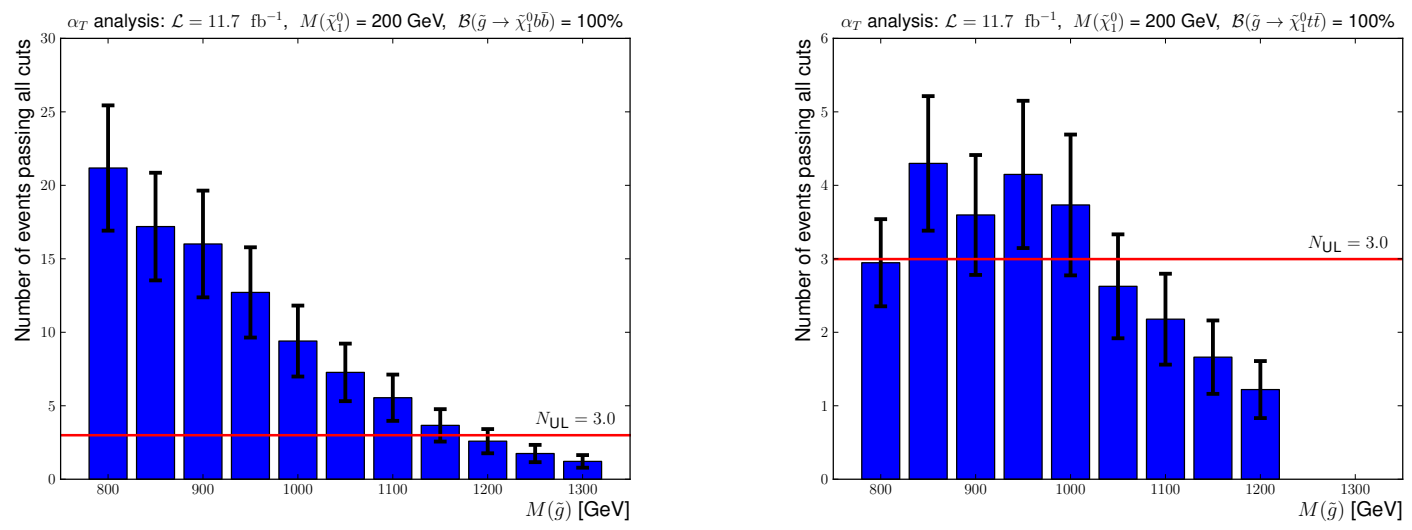

Figure 5: Validation of CMS analysis [6] for the simplified SUSY scenarios, $\tilde{g} \rightarrow b \bar{b} \tilde{\chi}_{1}^{0}$ (left) and $\tilde{g} \rightarrow t \bar{t} \tilde{\chi}_{1}^{0}$ (right). The CMS analysis rules out gluinos lighter than $1160 \mathrm{GeV}$ in the T1bbbb model and $1025 \mathrm{GeV}$ in the T1tttt simplified models. Our results are in excellent agreement with the CMS bounds.

observed number of events and the expected SM background. These bounds are indicated in Fig. 5 by the horizontal red line annotated by $N_{\mathrm{UL}}$ for an LSP of mass $200 \mathrm{GeV}$, which is the region most relevant for our model. The bounds on the gluino mass obtained from our (the CMS) analysis are 1130 (1125) GeV in the T1bbbb model and 975 (950) GeV in the T1tttt model. The values obtained in the validation are in excellent agreement with those in the CMS analysis [6].

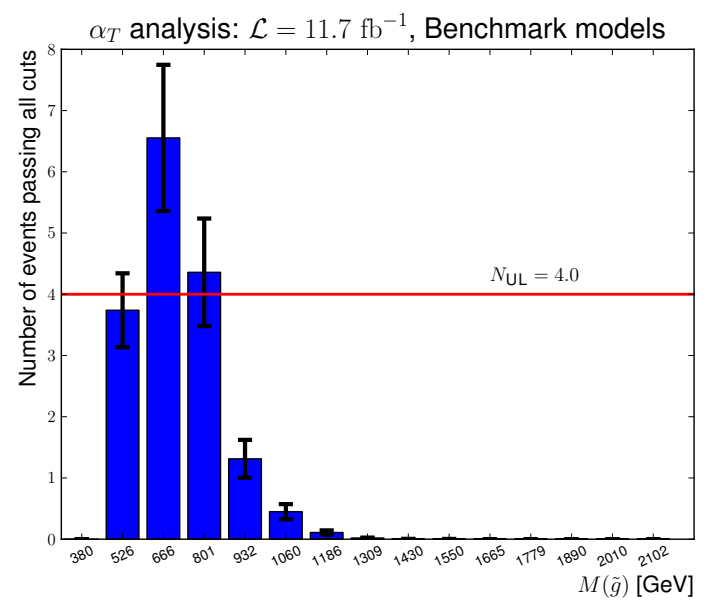

Figure 6: The number of events passing all cuts corresponding to the bin with 4 or more jets, 2 b-jets, and $775<H_{T}(\mathrm{GeV})<875$ in Ref.[6] for the benchmark models. The labels on the $x$-axis denote the gluino mass in each benchmark model. The red horizontal line shows the $95 \%$ upper limit on the allowed events from new physics. The error bars are derived from the uncertainty in estimating the NLO gluino production cross-section. 
We find the bins described in Tab. 4 of this paper to be the most constraining. Note that these bins select a particular number of b-jets. In the benchmark models, the gluinos have a significant branching ratio to a gluon and a neutralino. Since this decay mode does not result in b-jets, the benchmark models are not well approximated by the simplified models for this analysis. We typically find that the benchmark models give fewer b-jets. Thus the simplified models and the benchmark models do not receive the strongest constraints from the same bin.

After successfully validating our analysis, we now move on to analyze our benchmark models. The number of events that pass the cuts for each benchmark model is shown in Fig. 6. The bounds are clearly much weaker for the benchmark models. While the T1bbbb (T1tttt) simplified models rule out gluinos with mass 1125 (950) $\mathrm{GeV}$, we rule out only gluinos with mass $\approx 775 \mathrm{GeV}$ for this analysis.

\section{$5.2 \Delta \hat{\phi}$ Analysis}

In Ref. [4], the CMS collaboration performed a search for gluinos decaying according to one of the simplified SUSY scenarios shown in Fig. 2 by looking for events with large transverse missing energy, jets and b-jets, and with no isolated electrons or muons. The data sample used in this analysis was recorded at $8 \mathrm{TeV}$ center of mass energy and includes $19.4 \mathrm{fb}^{-1}$ of data. The analysis required at least 3 jets (and at least 1 b-tagged jet) with $p_{T}>50 \mathrm{GeV}$ and $|\eta|<2.4$, and binned the events into 14 signal regions with different ranges of $\mathbb{E}_{T}, H_{T}$ and $N_{\text {b-jet }}$. The details of the binning can be found in Tab. 1 and Tab. 2 of Ref. [4]. For neutralino masses of $0-400 \mathrm{GeV}$, we find the signal region summarized in the last row of Tab. 4 to be the most constraining one. In addition, events are required to have $\Delta \hat{\phi}_{\min }>4.0$, where $\Delta \hat{\phi}_{\min }=\min \left(\Delta \phi_{i} / \sigma_{\Delta \phi_{i}}\right)$ and $\Delta \phi$ is the angle between a jet and the negative of the $\mathbb{E}_{T}$ vector, and $\sigma_{\Delta \phi_{i}}$ is the estimated resolution of $\Delta \phi$. More on this observable can be found in Ref. [21]. QCD background events are characterized by low $\Delta \phi$, since the $p_{T}$ mis-measurement gives rise to most of the missing energy in a QCD event. By requiring $\Delta \hat{\phi}_{\min }>4.0$, most of the QCD backgrounds are eliminated.

As with the previous analyses, we first validate the CMS analysis. For this analysis, CMS quotes that the nominal b-tagging efficiency is $75 \%$ for jets with a $p_{T}$ value of $80 \mathrm{GeV}^{8}$. We adapt the Delphes CMS card using the following information from the CMS analysis: b-tagging efficiency, electron, and muon isolation criteria, and charged track definition and isolation criteria. Using the information on the number of observed events and the expected Standard Model background from Ref. [4] we determine bounds for the simplified models T1tttt and T1bbbb. The result is shown in Fig. 7. The bounds on the gluino mass obtained from our validation analysis (CMS analysis) are 1250 (1170) GeV in the T1bbbb model and 1100 (1020) GeV in the T1tttt simplified model. The agreement between the two quoted numbers is within the expectations from the tools that we have used.

We now proceed to show the results of this analysis, interpreted for the benchmark models discussed in Section 2. We generate 10,000 events for each of the benchmark models and apply the same cuts, and since these benchmark models do not fall into either of the simplified scenarios, we expect the bounds on the benchmark models to be weaker. In many cases, especially when the mass of the gluino is greater than $1 \mathrm{TeV}$, loop decays to a gluon and a neutralino are enhanced and the final

\footnotetext{
${ }^{8}$ We thank Mike Saelim for a digitized version of the b-tagging efficiency from Ref. [22].
} 

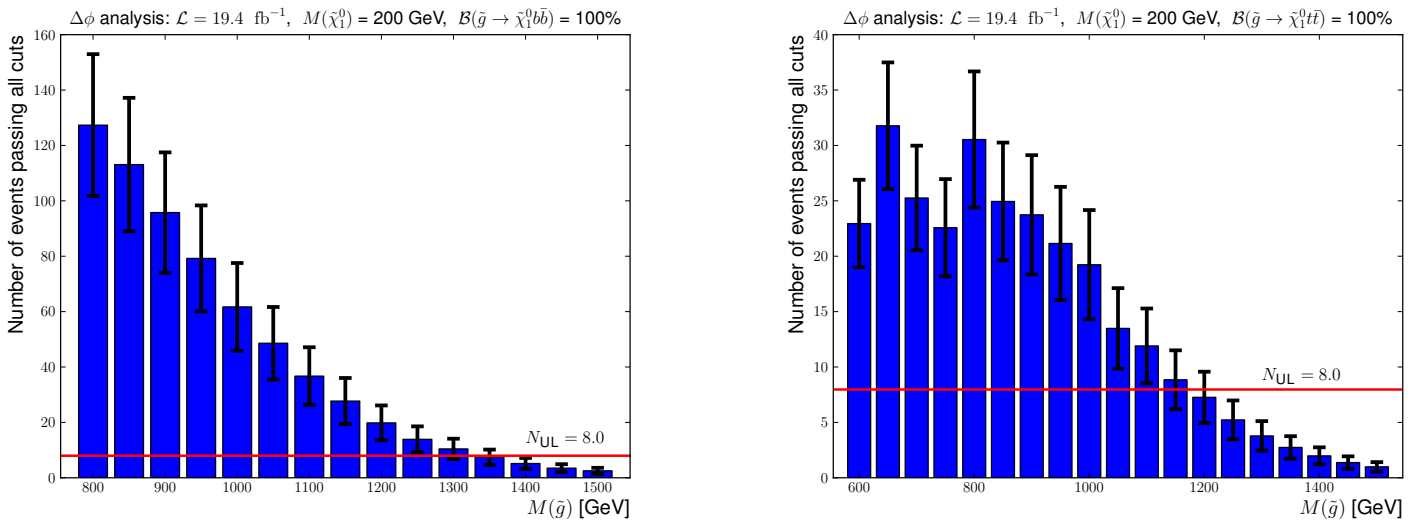

Figure 7: Validation of CMS analysis [4] for the simplified SUSY scenarios, $\tilde{g} \rightarrow \tilde{\chi}_{1}^{0} b \bar{b}$ (left) and $\tilde{g} \rightarrow \tilde{\chi}_{1}^{0} t \bar{t}$ (right). The CMS analysis rules out gluinos lighter than $1170 \mathrm{GeV}$ in the T1bbbb model and $1020 \mathrm{GeV}$ in the T1tttt simplified models. Our results are in agreement with the CMS bounds within the expectations of our numerical tools.

states will not have b-tagged jets. The number of events that pass the cuts for each benchmark model is shown in Fig. 8. It is clear from the figure that fewer events pass the cuts, since the final states from the gluino decays do not come from a single decay topology and there are, on average, fewer b-jets. Thus, in comparison with the simplified models, where we were able to rule out gluinos of $1100 \mathrm{GeV}$, we rule out gluinos only lighter than $\approx 1000 \mathrm{GeV}$ with this analysis.

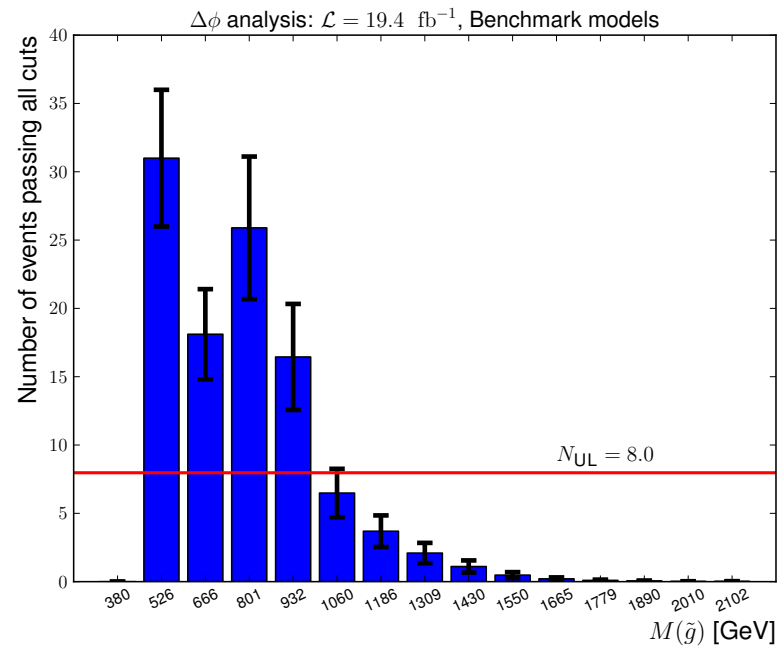

Figure 8: The number of events passing all cuts described in Tab. 4 for the benchmark models. The labels on the $x$-axis denote the gluino mass in each benchmark model. The red horizontal line shows the $95 \%$ upper limit on the allowed events from new physics. The error bars are derived from the uncertainty in estimating the NLO gluino production cross-section. 


\section{Conclusions}

In this paper, we studied the LHC phenomenology of Yukawa unified SO(10) models. From a wide range of available experimental analyses, we picked those that are most applicable to our case: The same sign di-lepton analysis [5] and two hadronic analyses, namely $\alpha_{T}[6]$ and $\Delta \hat{\phi}[4]$. These analyses are based on simplified models with typically only one decay channel for the gluino, and we re-interpreted their results to set limits on Yukawa unified $\mathrm{SO}(10)$ models.

The first question we addressed was: Which models do survive? To that end, we picked 15 benchmark models strategically placed along the $\chi^{2}$ curve that constitutes the best fit of Yukawa unified $\mathrm{SO}(10)$ models to low-energy data (cf. Fig. 1 and Tab. 2), and then looked at the signal events that pass all cuts for the respective analysis. We found that most of the benchmark models with a gluino mass of approximately $1000 \mathrm{GeV}$ or greater survive and cannot be ruled out with current data (cf. Fig. 4(b), Fig. 6, Fig. 8). Hence many of our benchmark points, Tab. 2, are not ruled out by the present LHC data. In particular, benchmark points YUc - YUf (Tab. 2) are still viable models which can be tested at LHC 14. As an important side remark, we mention that before analyzing the benchmark models, we validated our analysis by reproducing the results for the simplified models. In all cases, the agreement was within $20 \%$, and by common lore, this is as close as we might expect to get to the results produced by the experimental collaborations with their more elaborate tools. This constitutes a non-trivial cross-check of our analysis procedure outlined in Fig. 3.

Another question we addressed was the dichotomy between benchmark and simplified models: How well do simplified models describe the Yukawa unified ones? Simplified models are designed to capture the main features of specific scenarios at the cost of (possibly over-)simplifying assumptions. Our findings indicate that for the same sign dilepton analysis, the simplified models describe our benchmark scanarios pretty well, but for the two hadronic analyses, the agreement is less pronounced. The reason is that for the benchmark models, there is a sizeable branching fraction for loop decays of the gluino to a gluon and a neutralino. However, the simplified models studied so far assume that all decays of the gluino are accompanied by b-quarks. As a consequence, the limits on the gluino mass that we obtain for the benchmark models are weaker than those in the case of the simplified scenarios.

One could argue that the bounds on the gluino mass obtained from simplified and benchmark models differ by less than $20 \%$ and are thus within the expected precision. However, the excellent agreement between the gluino bounds from Refs. [4, 6] and our validation (cf. Fig. 5 and Fig. 7) encourages us to believe that this is indeed a physical effect and not a relic of our analysis procedure. In other words, for the hadronic analyses, the agreement in the validation of the benchmark models is about $8-10 \%$, whereas the bounds we obtain for the benchmark models from the same analysis is less by about $20 \%$. That is why we conclude that our gluino bounds are weaker.

Lastly, we would like to point out that the $\chi^{2}$ analysis of Yukawa unified $\mathrm{SO}(10)$ models suggests an upper bound on the gluino mass of about $3 \mathrm{TeV}$ (for $m_{16} \lesssim 30 \mathrm{TeV}$ ). Since the amount of fine-tuning increases with $m_{16}$, larger values of the gluino mass are theoretically disfavored. In addition, in our previous paper, Ref. [1], we showed that $\chi^{2}$ was minimized for both the three family and third family analysis at $m_{16}=20 \mathrm{TeV}$ with an upper bound $m_{\tilde{g}} \leq 2 \mathrm{TeV}$ (see also Fig. 1). For larger $m_{16}$, the pull to $\chi^{2}$ for $M_{W}$ increased. ${ }^{9}$ The LHC, after collecting $300 \mathrm{fb}^{-1}$ of data at $14 \mathrm{TeV}$, has

\footnotetext{
${ }^{9} \mathrm{~A}$ similar result was found in Ref. [23].
} 
a $5 \sigma$ discovery potential for gluinos with mass $\lesssim 1.9 \mathrm{TeV}[24,25]$. Thus in the most favored range of parameters the gluino should be observed at the LHC $14 \mathrm{TeV}$. Finally, Yukawa unified models make other significant predictions for the SUSY particle spectrum as can be seen for our benchmark models (Tab. 2).

\section{Acknowledgments}

We acknowledge useful discussions with Sabine Kraml (Grenoble), Sezen Sekmen (Florida State), Andre Lessa (Sao Paulo), Joerg Meyer (Karlsruhe), Noel Dawe (Vancouver/CERN), Peter Waller (Manchester) and Radovan Dermisek (Indiana). A.A. and S.R. received partial support for this work from DOE/ER/01545-90. A.A. and A.W. acknowledge partial support from LabEx ENIGMASS, and A.A. would like to thank the LPSC Grenoble where part of this work was completed for their hospitality. We thank the Ohio Supercomputer Center and the Centre de Calcul de l'Institut National de Physique Nucléaire et Physique des Particules in Lyon for using their resources. S.R. thanks the Galileo Galilei Institute for Theoretical Physics for the hospitality and the INFN for partial support and CETUP 2013 for their hospitality and partial support during the completion of this work. S.R. would also like to thank CETUP* (Center for Theoretical Underground Physics and Related Areas), supported by the US Department of Energy under Grant No. DE-SC0010137 and by the US National Science Foundation under Grant No. PHY-1342611, for its hospitality and partial support during the 2013 Summer Program. 


\section{A Upper bound on gluino mass}

Note, in Fig. 9 we take $m_{16}=30 \mathrm{TeV}$. We find that the upper bound on the gluino mass increases as $m_{16}$ increases. This is due to the combination of the constraints from the bottom quark and light Higgs mass. For $m_{16}=30 \mathrm{TeV}$ the upper bound on the gluino mass at $90 \% \mathrm{CL}$ is $2.85 \mathrm{TeV}$. Although in our three family analysis $\chi^{2}$ increases as $m_{16}$ increases, we do not believe that there is a strict upper bound on $m_{16}$ other than the fact that the level of fine-tuning increases as $m_{16}$ increases.

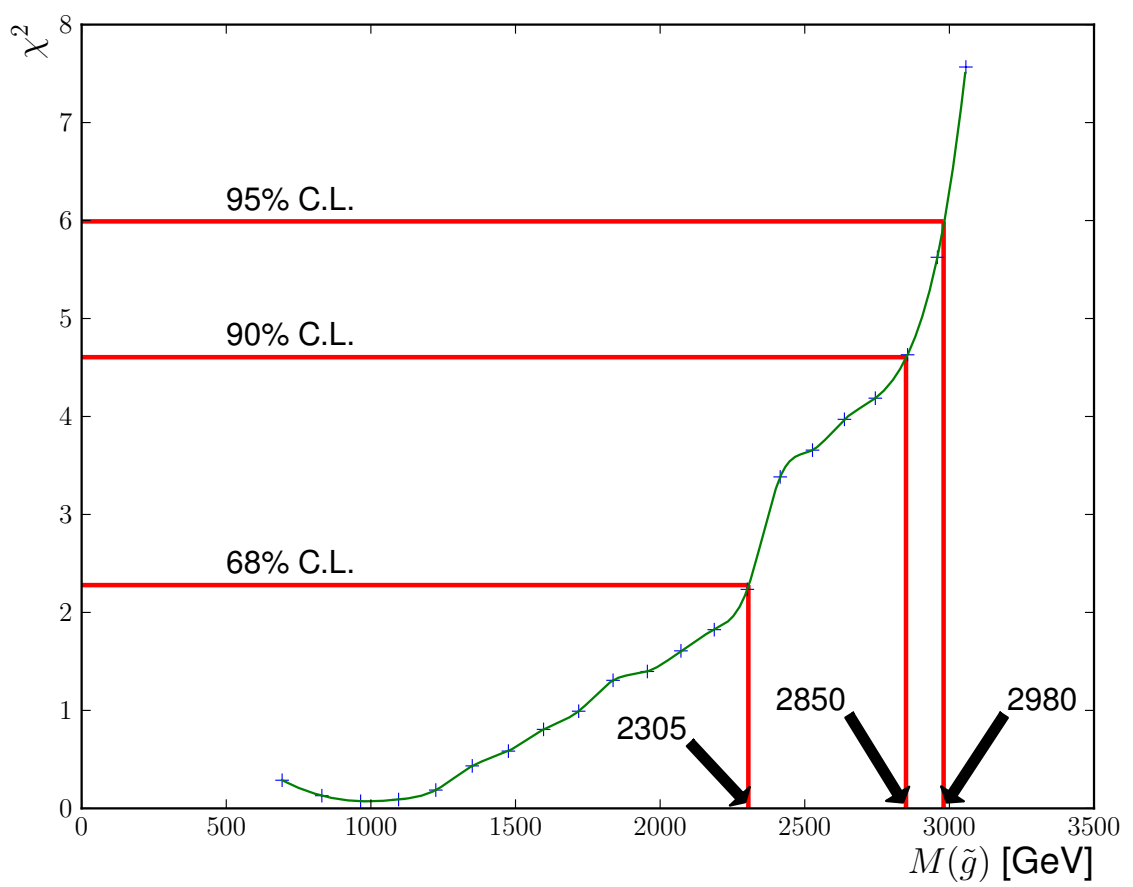

Figure 9: The $\chi^{2}$ function for fixed $m_{16}=30 \mathrm{TeV}$ and different values of $M_{1 / 2}$ corresponding to the gluino masses indicated on the $x$-axis. In minimizing $\chi^{2}$ for each point, we followed the same procedure as in Ref. [1] except that we now do not penalize for gluino masses smaller than a certain lower bound. The confidence level intervals correspond to the $\chi^{2}$ distribution with two degrees of freedom. Note, the $90 \%$ upper bound on the gluino mass increases as $m_{16}$ increases. 


\section{B Details on the Benchmark Models}

Table 5: Benchmark model YUa

$\left(1 / \alpha_{G}, M_{G}, \epsilon_{3}, \lambda\right)=\left(25.95,2.87 \times 10^{16} \mathrm{GeV},-1.04 \%, 0.603\right)$, $\left(m_{16}, M_{1 / 2}, A_{0}, \mu\left(M_{Z}\right)\right)=(20000,150,-41289,869) \mathrm{GeV}$, $\left(\left(m_{H_{d}} / m_{16}\right)^{2},\left(m_{H_{u}} / m_{16}\right)^{2}, \tan \beta\right)=(1.87,1.62,49.69)$

\begin{tabular}{|l|c|c|c|c|}
\hline Observable & Fit value & Experimental Value & Pull & Error \\
\hline$M_{Z}$ & 91.1876 & 91.1876 & 0.0000 & 0.4559 \\
$M_{W}$ & 80.5166 & 80.4360 & 0.2004 & 0.4025 \\
$1 / \alpha_{e m}$ & 137.1232 & 137.0360 & 0.1272 & 0.0000 \\
$G_{\mu} \times 10^{5}$ & 1.1689 & 1.1664 & 0.2172 & 0.0117 \\
$\alpha_{3}$ & 0.1184 & 0.1184 & 0.0337 & 0.0009 \\
\hline$M_{t}$ & 173.5973 & 173.5000 & 0.0735 & 1.3238 \\
$m_{b}\left(m_{b}\right)$ & 4.1815 & 4.1800 & 0.0403 & 0.0366 \\
$m_{\tau}$ & 1.7766 & 1.7768 & 0.0242 & 0.0089 \\
\hline$M_{h}$ & 124.33 & 125.30 & 0.3168 & 3.0676 \\
\hline $\mathcal{B}\left(B \rightarrow X_{s} \gamma\right) \times 10^{4}$ & 3.2670 & 3.4300 & 0.0995 & 1.6374 \\
$\mathcal{B}\left(B_{s} \rightarrow \mu^{+} \mu^{-}\right) \times 10^{9}$ & 3.1369 & 3.2000 & 0.0387 & 1.6308 \\
\hline Total $\chi^{2}$ & \multicolumn{5}{|c|}{$\mathbf{0 . 2 2 4 0 1 4}$} & \\
\hline
\end{tabular}

Table 6: Benchmark model YUb

$\left(1 / \alpha_{G}, M_{G}, \epsilon_{3}, \lambda\right)=\left(26.00,2.71 \times 10^{16} \mathrm{GeV},-0.94 \%, 0.603\right)$, $\left(m_{16}, M_{1 / 2}, A_{0}, \mu\left(M_{Z}\right)\right)=(20000,200,-41272,889) \mathrm{GeV}$, $\left(\left(m_{H_{d}} / m_{16}\right)^{2},\left(m_{H_{u}} / m_{16}\right)^{2}, \tan \beta\right)=(1.87,1.62,49.69)$

\begin{tabular}{|l|c|c|c|c|}
\hline Observable & Fit value & Experimental Value & Pull & Error \\
\hline$M_{Z}$ & 91.1876 & 91.1876 & 0.0000 & 0.4559 \\
$M_{W}$ & 80.5133 & 80.4360 & 0.1920 & 0.4025 \\
$1 / \alpha_{e m}$ & 137.1111 & 137.0360 & 0.1096 & 0.0000 \\
$G_{\mu} \times 10^{5}$ & 1.1688 & 1.1664 & 0.2047 & 0.0117 \\
$\alpha_{3}$ & 0.1184 & 0.1184 & 0.0523 & 0.0009 \\
\hline$M_{t}$ & 173.5907 & 173.5000 & 0.0685 & 1.3238 \\
$m_{b}\left(m_{b}\right)$ & 4.1817 & 4.1800 & 0.0478 & 0.0366 \\
$m_{\tau}$ & 1.7766 & 1.7768 & 0.0302 & 0.0089 \\
\hline$M_{h}$ & 123.58 & 125.30 & 0.5602 & 3.0676 \\
\hline $\mathcal{B}\left(B \rightarrow X_{s} \gamma\right) \times 10^{4}$ & 3.2584 & 3.4300 & 0.1048 & 1.6374 \\
$\mathcal{B}\left(B_{s} \rightarrow \mu^{+} \mu^{-}\right) \times 10^{9}$ & 3.1384 & 3.2000 & 0.0378 & 1.6308 \\
\hline Total $\chi^{2}$ & \multicolumn{5}{|l|}{$\mathbf{0 . 4 2 7 6 5 8}$} & \\
\hline
\end{tabular}


Table 7: Benchmark model YUc

$\left(1 / \alpha_{G}, M_{G}, \epsilon_{3}, \lambda\right)=\left(25.97,2.88 \times 10^{16} \mathrm{GeV},-1.30 \%, 0.605\right)$,

$\left(m_{16}, M_{1 / 2}, A_{0}, \mu\left(M_{Z}\right)\right)=(20000,250,-41231,824) \mathrm{GeV}$,

$\left(\left(m_{H_{d}} / m_{16}\right)^{2},\left(m_{H_{u}} / m_{16}\right)^{2}, \tan \beta\right)=(1.87,1.62,49.68)$

\begin{tabular}{|c|c|c|c|c|}
\hline Observable & Fit value & Experimental Value & Pull & Error \\
\hline$M_{Z}$ & 91.1876 & 91.1876 & 0.0000 & 0.4559 \\
\hline$M_{W}$ & 80.5329 & 80.4360 & 0.2407 & 0.4025 \\
\hline $1 / \alpha_{e m}$ & 137.1585 & 137.0360 & 0.1786 & 0.0000 \\
\hline$G_{\mu} \times 10^{5}$ & 1.1694 & 1.1664 & 0.2629 & 0.0117 \\
\hline$\alpha_{3}$ & 0.1183 & 0.1184 & 0.0758 & 0.0009 \\
\hline$M_{t}$ & 173.6810 & 173.5000 & 0.1367 & 1.3238 \\
\hline$m_{b}\left(m_{b}\right)$ & 4.1827 & 4.1800 & 0.0750 & 0.0366 \\
\hline$m_{\tau}$ & 1.7763 & 1.7768 & 0.0532 & 0.0089 \\
\hline$M_{h}$ & 123.01 & 125.30 & 0.7474 & 3.0676 \\
\hline $\mathcal{B}\left(B \rightarrow X_{s} \gamma\right) \times 10^{4}$ & 3.2279 & 3.4300 & 0.1234 & 1.6374 \\
\hline $\mathcal{B}\left(B_{s} \rightarrow \mu^{+} \mu^{-}\right) \times 10^{9}$ & 3.1347 & 3.2000 & 0.0400 & 1.6308 \\
\hline \multicolumn{4}{|l|}{ Total $\chi^{2}$} & \\
\hline
\end{tabular}

Table 8: Benchmark model YUd

$\left(1 / \alpha_{G}, M_{G}, \epsilon_{3}, \lambda\right)=\left(25.97,2.89 \times 10^{16} \mathrm{GeV},-1.44 \%, 0.605\right)$,

$\left(m_{16}, M_{1 / 2}, A_{0}, \mu\left(M_{Z}\right)\right)=(20000,300,-41171,878) \mathrm{GeV}$,

$\left(\left(m_{H_{d}} / m_{16}\right)^{2},\left(m_{H_{u}} / m_{16}\right)^{2}, \tan \beta\right)=(1.87,1.62,49.67)$

\begin{tabular}{|l|c|c|c|c|}
\hline Observable & Fit value & Experimental Value & Pull & Error \\
\hline$M_{Z}$ & 91.1876 & 91.1876 & 0.0000 & 0.4559 \\
$M_{W}$ & 80.5432 & 80.4360 & 0.2664 & 0.4025 \\
$1 / \alpha_{e m}$ & 137.1270 & 137.0360 & 0.1327 & 0.0000 \\
$G_{\mu} \times 10^{5}$ & 1.1704 & 1.1664 & 0.3426 & 0.0117 \\
$\alpha_{3}$ & 0.1183 & 0.1184 & 0.1036 & 0.0009 \\
\hline$M_{t}$ & 173.7543 & 173.5000 & 0.1921 & 1.3238 \\
$m_{b}\left(m_{b}\right)$ & 4.1837 & 4.1800 & 0.1014 & 0.0366 \\
$m_{\tau}$ & 1.7762 & 1.7768 & 0.0682 & 0.0089 \\
\hline$M_{h}$ & 122.41 & 125.30 & 0.9412 & 3.0676 \\
\hline $\mathcal{B}\left(B \rightarrow X_{s} \gamma\right) \times 10^{4}$ & 3.2293 & 3.4300 & 0.1226 & 1.6374 \\
$\mathcal{B}\left(B_{s} \rightarrow \mu^{+} \mu^{-}\right) \times 10^{9}$ & 3.1325 & 3.2000 & 0.0414 & 1.6308 \\
\hline Total $\chi^{2}$ & \multicolumn{4}{|l|l|l|}{} \\
\hline
\end{tabular}


Table 9: Benchmark model YUe

$\left(1 / \alpha_{G}, M_{G}, \epsilon_{3}, \lambda\right)=\left(25.99,2.80 \times 10^{16} \mathrm{GeV},-1.57 \%, 0.603\right)$,

$\left(m_{16}, M_{1 / 2}, A_{0}, \mu\left(M_{Z}\right)\right)=(20000,400,-41088,975) \mathrm{GeV}$,

$\left(\left(m_{H_{d}} / m_{16}\right)^{2},\left(m_{H_{u}} / m_{16}\right)^{2}, \tan \beta\right)=(1.88,1.62,49.62)$

\begin{tabular}{|l|c|c|c|c|}
\hline$M_{Z}$ & 91.1876 & 91.1876 & 0.0000 & 0.4559 \\
$M_{W}$ & 80.5546 & 80.4360 & 0.2946 & 0.4025 \\
$1 / \alpha_{e m}$ & 137.0434 & 137.0360 & 0.0109 & 0.0000 \\
$G_{\mu} \times 10^{5}$ & 1.1718 & 1.1664 & 0.4629 & 0.0117 \\
$\alpha_{3}$ & 0.1182 & 0.1184 & 0.1644 & 0.0009 \\
\hline$M_{t}$ & 173.8117 & 173.5000 & 0.2355 & 1.3238 \\
$m_{b}\left(m_{b}\right)$ & 4.1840 & 4.1800 & 0.1098 & 0.0366 \\
$m_{\tau}$ & 1.7761 & 1.7768 & 0.0797 & 0.0089 \\
\hline$M_{h}$ & 121.24 & 125.30 & 1.3219 & 3.0676 \\
\hline $\mathcal{B}\left(B \rightarrow X_{s} \gamma\right) \times 10^{4}$ & 3.2057 & 3.4300 & 0.1370 & 1.6374 \\
$\mathcal{B}\left(B_{s} \rightarrow \mu^{+} \mu^{-}\right) \times 10^{9}$ & 3.6184 & 3.2000 & 0.2566 & 1.6308 \\
\hline Total $\chi^{2}$ & \multicolumn{3}{|c|}{$\mathbf{2 . 2 3 4 1 3}$} & \\
\hline
\end{tabular}

Table 10: Benchmark model YUf

$\left(1 / \alpha_{G}, M_{G}, \epsilon_{3}, \lambda\right)=\left(26,2.5 \times 10^{16} \mathrm{GeV},-1.16 \%, 0.597\right)$, $\left(m_{16}, M_{1 / 2}, A_{0}, \mu\left(M_{Z}\right)\right)=(20000,600,-41221,664) \mathrm{GeV}$, $\left(\left(m_{H_{d}} / m_{16}\right)^{2},\left(m_{H_{u}} / m_{16}\right)^{2}, \tan \beta\right)=(1.87,1.62,49.24)$

\begin{tabular}{|l|c|c|c|c|}
\hline$M_{Z}$ & 91.1876 & 91.1876 & 0.0000 & 0.4559 \\
$M_{W}$ & 80.5577 & 80.4360 & 0.3025 & 0.4025 \\
$1 / \alpha_{e m}$ & 136.8484 & 137.0360 & 0.2742 & 0.0000 \\
$G_{\mu} \times 10^{5}$ & 1.1729 & 1.1664 & 0.5616 & 0.0117 \\
$\alpha_{3}$ & 0.1183 & 0.1184 & 0.1345 & 0.0009 \\
\hline$M_{t}$ & 173.6236 & 173.5000 & 0.0934 & 1.3238 \\
$m_{b}\left(m_{b}\right)$ & 4.1870 & 4.1800 & 0.1904 & 0.0366 \\
$m_{\tau}$ & 1.7757 & 1.7768 & 0.1234 & 0.0089 \\
\hline$M_{h}$ & 119.65 & 125.30 & 1.8411 & 3.0676 \\
\hline$B R\left(B \rightarrow X_{s} \gamma\right) \times 10^{4}$ & 3.1850 & 3.4300 & 0.1496 & 1.6374 \\
$B R\left(B_{s} \rightarrow \mu^{+} \mu^{-}\right) \times 10^{9}$ & 3.1953 & 3.2000 & 0.0029 & 1.6308 \\
\hline Total $\chi^{2}$ & & $\mathbf{3 . 9 7 2 4 8}$ & \\
\hline
\end{tabular}

\section{References}

[1] A. Anandakrishnan, S. Raby, and A. Wingerter, "Yukawa Unification Predictions for the LHC," Phys.Rev. D87 (2013) 055005, 1212.0542.

[2] R. Dermisek, S. Raby, L. Roszkowski, and R. Ruiz de Austri, "Dark matter and $B_{s} \rightarrow \mu^{+} \mu^{-}$ with minimal SO(10) soft SUSY breaking II," JHEP 0509 (2005) 029, hep-ph/0507233. 
[3] M. Albrecht, W. Altmannshofer, A. J. Buras, D. Guadagnoli, and D. M. Straub, "Challenging $\mathrm{SO}(10)$ SUSY GUTs with family symmetries through FCNC processes," JHEP 0710 (2007) 055, 0707.3954.

[4] CMS Collaboration Collaboration, S. Chatrchyan et al., "Search for gluino mediated bottom- and top-squark production in multijet final states in pp collisions at $8 \mathrm{TeV}$," 1305.2390.

[5] CMS Collaboration Collaboration, S. Chatrchyan et al., "Search for new physics in events with same-sign dileptons and $b$ jets in $p p$ collisions at $\sqrt{s}=8 \mathrm{TeV}, " J H E P 1303$ (2013) 037, 1212.6194.

[6] CMS Collaboration Collaboration, S. Chatrchyan et al., "Search for supersymmetry in hadronic final states with missing transverse energy using the variables $\alpha_{T}$ and b-quark multiplicity in pp collisions at $\sqrt{s}=8 \mathrm{TeV}, " 1303.2985$.

[7] ATLAS Collaboration, "Search for strongly produced superpartners in final states with two same sign leptons with the atlas detector using $21 \mathrm{fb}-1$ of proton-proton collisions at sqrt(s)=8 tev.." http://cds. cern.ch/record/1522430/files/ATLAS-CONF-2013-007.pdf, Mar, 2013. ATLAS-CONF-2013-007.

[8] ATLAS Collaboration, "Search for new phenomena using final states with large jet multiplicities and missing transverse momentum with atlas in $20 \mathrm{fb}^{-1}$ of $\sqrt{s}=8$ tev proton-proton collisions." http://cds.cern.ch/record/1547571/files/ATLAS-CONF-2013-054.pdf, May, 2013. ATLAS-CONF-2013-054.

[9] ATLAS Collaboration, "Search for squarks and gluinos with the atlas detector in final states with jets and missing transverse momentum and $20.3 \mathrm{fb}^{-1}$ of $\sqrt{s}=8$ tev proton-proton collision data." http://cds. cern.ch/record/1547563/files/ATLAS-CONF-2013-047.pdf, May, 2013. ATLAS-CONF-2013-047.

[10] M. Kramer, A. Kulesza, R. van der Leeuw, M. Mangano, S. Padhi, et al., "Supersymmetry production cross sections in $p p$ collisions at $\sqrt{s}=7 \mathrm{TeV}, " 1206.2892$.

[11] M. Muhlleitner, A. Djouadi, and Y. Mambrini, "SDECAY: A Fortran code for the decays of the supersymmetric particles in the MSSM," Comput.Phys.Commun. 168 (2005) 46-70, hep-ph/0311167.

[12] H. Baer, X. Tata, and J. Woodside, "PHENOMENOLOGY OF GLUINO DECAYS VIA LOOPS AND TOP QUARK YUKAWA COUPLING," Phys.Rev. D42 (1990) 1568-1576.

[13] H. Baer, C.-h. Chen, M. Drees, F. Paige, and X. Tata, "Collider phenomenology for supersymmetry with large tan Beta," Phys.Rev.Lett. 79 (1997) 986-989, hep-ph/9704457.

[14] H. Baer, C.-h. Chen, M. Drees, F. Paige, and X. Tata, "Supersymmetry reach of Tevatron upgrades: The Large tan Beta case," Phys.Rev. D58 (1998) 075008, hep-ph/9802441.

[15] T. Sjostrand, S. Mrenna, and P. Z. Skands, "A Brief Introduction to PYTHIA 8.1," Comput.Phys.Commun. 178 (2008) 852-867, 0710.3820. 
[16] M. Dobbs and J. B. Hansen, "The HepMC C++ Monte Carlo event record for High Energy Physics," Comput.Phys.Commun. 134 (2001) 41-46.

[17] S. Ovyn, X. Rouby, and V. Lemaitre, "DELPHES, a framework for fast simulation of a generic collider experiment," 0903.2225.

[18] R. Brun and F. Rademakers, "ROOT: An object oriented data analysis framework," Nucl. Instrum. Meth. A389 (1997) 81-86.

[19] W. Beenakker, R. Hopker, and M. Spira, "PROSPINO: A Program for the production of supersymmetric particles in next-to-leading order QCD," hep-ph/9611232.

[20] J. D. Hunter, "Matplotlib: A 2d graphics environment," Computing In Science E Engineering 9 (2007), no. 3, 90-95.

[21] CMS Collaboration Collaboration, S. Chatrchyan et al., "Search for supersymmetry in events with b-quark jets and missing transverse energy in pp collisions at $7 \mathrm{TeV}$," Phys.Rev. D86 (2012) 072010, 1208.4859.

[22] CMS Collaboration Collaboration, S. Chatrchyan et al., "Identification of b-quark jets with the CMS experiment," JINST 8 (2013) P04013, 1211.4462.

[23] V. Barger, P. Huang, M. Ishida, and W.-Y. Keung, "Scalar-Top Masses from SUSY Loops with $125 \mathrm{GeV}$ mh and Precise Mw," Phys.Lett. B718 (2013) 1024-1030, 1206.1777.

[24] H. Baer, V. Barger, A. Lessa, and X. Tata, "Discovery potential for SUSY at a high luminosity upgrade of LHC14," Phys.Rev. D86 (2012) 117701, 1207.4846.

[25] CMS Collaboration Collaboration, "Projected Performance of an Upgraded CMS Detector at the LHC and HL-LHC: Contribution to the Snowmass Process," 1307.7135. 